\title{
Research on Evolution Model and Crisis Prevention and Control of New Media Public Opinion
}

\author{
Kongyu Yang, Ruijie Miao \\ School of Information Management, Beijing Information Since and Technology University \\ Beijing, 100192 \\ Email: kyyang@bistu.edu.cn
}

\begin{abstract}
This paper simulates the multi-layer defense mechanism of biological immune system and proposes a new media public opinion evolution model applicable to the evolution of public opinions of emergencies and crisis prevention and control, including acquisition layer, analysis layer, response layer, disposal layer and recovery layer. By integrating a variety of public opinion monitoring and analysis technologies, equipment and software development, and then designs a public opinion crisis prevention and control prototype system with adaptive evolutionary capabilities, which is multi-layer linkage combined with public opinion monitoring, public opinion detection, public opinion early warning, public opinion decision-making and public opinion situation recovery.
\end{abstract}

Keywords-Artificial immune system; Public opinion evolution model; Public opinion detection; Public opinion early warning; Public opinion crisis prevention and control

\section{INTRODUCTION}

In August 2017, the latest semi-annual report released by the China Internet Network Information Center showed, as of June this year, the number of netizens in China reached 751 million, accounting for $1 / 5$ of the total number of Internet users in the world. China's Internet penetration rate reached $54.3 \%$, of which mobile phone users reached 724 million, the use of mobile Internet access rate as high as $96.3 \%$. The proportion of mobile Internet is rising based on the high base. With the growth of netizens, people express different opinions by using the new media and their consciousness and enthusiasm for participating in public affairs are rising day by day. Consequently, online speech has reached an unprecedented level of activity. People express their views and spread ideas through the Internet in the domestic or international important events, which can immediately form the online public opinion, and then produce enormous pressure of public opinion. And these can not be ignored by any department or institution. It can be said that the Internet has become the distribution center of ideological and cultural information and an amplifier of public opinion.

Meanwhile, new media platforms derived from instant messaging tools such as WeChat circle of friends and QQ space have $85.8 \%$ and $67.8 \%$ respectively of user usage. It can be seen that Chinese society has entered the Internet era, or rather, has entered a new media era in which broadband, mobile and social media are superimposed. The new media with features of network, digitization, real-time and mobile quickly affects and changes the evolution mode of public opinion, and makes public opinion communication more smooth and timely. At the same time, the sudden public opinion crisis will become more difficult to control.

At the academic seminar on "Public Opinion and New Media" at the end of 2012, aiming at the "new orientation of public opinion research under the background of new media", the relevant experts pointed out that the new media represented by Weibo has fundamentally changed the media ecological structure. Under the new media environment, once emergency events occur, it will cause the sudden change of network public opinion and set off a wave of public opinion, thus affecting the social stability and development. Since self-media such as Weibo has the advantages of instant, convenience, widespread usage and strong interactivity that other traditional media cannot match which, to a great extent, poses a serious challenge to social public security while bringing security risks of internet virtual society under the background of new media. Compared with the traditional media, the important feature of new media lies in the interactive mode of communication, which can prompt the rapid rise of public opinion. In order to avoid sharp deterioration of the situation, the government departments and relevant operator must rely on and grasp the law of the development and evolution of new media consensus, guide public opinion timely and establish effective crisis response and disposal mechanisms according to the specific conditions.

There will usually go through four development stages according to the network public opinion :the germination period - Weibo users break the sensitive topic; boom period traditional media, news websites follow up report; peak period - the interaction between new media and traditional media forms the resultant force of public opinion ; Remission period the government response ;Combined with the network of intelligent public opinion monitoring technology and tools in the new media environment, we must study and establish the evolutionary theoretical model of new media opinion in order to construct an effective public opinion monitoring and defense system based on the evolution of public opinion, then use information technology and intelligent method to develop and run public opinion crisis prevention and rapid emergency response system.

In recent years, although there has been more research on early warning of Internet public opinion crisis, at present there 
is a lack of guiding theoretical evolution model and defensive crisis warning system : 1 . Most of the early warning evaluation index system is mainly post-assessment (trend warning), lack of early warning (defensive warning) research of dealing with the network public opinion crises; 2 . The majority of literature on early warning indicators of public opinion on the Internet lacks necessary quantitative explanations and data acquisition methods for the final indicators, resulting in unconvincing index system; 3. Most of the indicators are qualitative description, but less quantitative description while the quantitative indicators make the judgment of public opinion information more objective and scientific.

In brief, the research on the public opinion prediction of new media represented by Weibo is more focused on public opinion discovery and text analysis based on Weibo short texts. However, the research on the public opinion evolution of new media and the prevention and control of crisis are not extensive.

\section{THE VALUE AND SIGNIFICANCE OF THE RESEARCH}

It can't be achieved by totally relying on manpower that real-time monitoring of the new media public opinion with the support of the cloud computing. Especially for emergencies, we not only need to conduct qualitative analysis and quantitative monitoring of network public opinion, but also need to get a more scientific and professional quick emergency solution. So, the use of information technology and intelligent means of network public opinion analysis and emergency response has become a top priority in this field. Therefore, it is urgent to research and design a kind of media public opinion forecasting and emergency response system which is based on real-time situations such as public opinion hot spots, netizens' opinions through the collection, classification, integration and screening of all kinds of information of new media. As the new media public opinion dissemination of the network often unexpectedly broke out in an unknown situation, completed quickly spread like a virus, which can easily lead to new social hot spots, often forming overlay effect of online and offline media. By contrast, we can find that this media spread and evolution process has a similarity with the virus defense immune processes that humans and organisms have.

The biological immune system is a necessary virus (antigen) monitoring and defense system for organism, especially human beings. It consists of immune function organs, tissues, antibody cells, immune effector molecules and other immune genes. Healthy individuals effectively protect themselves against external pathogens, harmful foreign body and its own cancercausing cells of the pathogenic factor (antigen), through the immune system's immune response, immune regulation, immune memory and immune surveillance mechanisms and immune evolution. The immune system includes nonspecific immunity and specific immunity. Specific immunity is a kind of adaptive complex system with intelligence and evolutionary ability, which is formed by the resistance of healthy individuals gradually accumulating when they resist the invasion of new germs. Just as the biological immune system, the process of public opinion evolution and crisis prevention includes several stages, such as obtaining information(perception of the corresponding antigen), analyzing information(antigen gene extraction), detecting sensitive words(matching of antibodies to antigens), monitoring the public opinion dynamically (generation and evolution of antibodies) and handling emergencies(clearance of antigens). The actual system will be fully realized from the media to obtain data, then analyse and extract antigen gene using data, thus realize the detection of antigen, promote the generation of antibody, achieve immunological learning and evolution of all immune processes, finish the acquisition of public opinion information (antigen), the extraction of sensitive words (antigenic genes), the analysis and detection (antibody matching) adaptive, resulting in forming decision support rules (antibody library) stored in data library or knowledge base, and provide flexible front-end active defense and automatic processing mechanism (immune defense), finally pass the contingency plan rapidly to decision maker for emergency management of the crisis. According to this, this project is going to create network public opinion evolution model with the immune function of active defense and autonomous emergency by studying the evolution of new media consensus and multi-layer defense system based on immune mechanism.

With the public opinion crisis in the new media environment is increasingly grim and the relevant research has been gradually deepened, we naturally think of the simulation of biological immune system on organism health monitoring and disease prevention, to build intelligent public opinion monitoring and emergency disposal system. Internet search engine can only provide related search services, to rely on artificial to active search. The results are motivated by business interests, and are heavily doped with unreasonable factors. And the accuracy and availability of the results are not strong enough, and the comprehensiveness and timeliness can not fully meet the needs of public opinion monitoring. Therefore, to establish a scientific network public opinion monitoring and emergency disposal system has become a research hotspot in academia. The existing public opinion monitoring system must reflect the dynamic process of decision-making, response and recovery on the basis of static collection and analysis. And then form the multi-layer immune defense system. Therefore, it is of high theoretical and practical value to study the intelligent evolution model of new media and the defense system of public opinion crisis with immune function by simulating biological immune system.

\section{THE MAIN CONTENT OF THE RESEARCH}

For practical applications, public opinion intelligent monitoring and prevention and control system will achieve the following specific functions: (1)Identify the hot topics and sensitive topics in time; (2)Realize propensity analysis; (3)Track the theme of public opinion; (4)Format the summary of public opinion automatically; (5)Identify the emergencies and their trend automatically; (6) Form trend analysis reports; (7)Realize the early warning of public opinion crises; (8)Achieve emergency response decision support according to the public opinion.

For this purpose, a successful public opinion monitoring and prevention system needs to dynamically monitor the public opinion information related to emergencies which is contained in or not included in the major media search engines. What's more, the system can also provide timely early warnings to 
major public opinions and provide quantitative statistical analysis data to support the prevention and control of public opinion, and assist in public opinion emergency response through automatic generation of public opinion reports.

\section{A. Research and establish Multi-layer evolution theoretical model based on new media communication platform}

Research and establish integrated public opinion monitoring system hierarchical model based on immune principles systematically which simulate multi-layer defense mechanism of biological immune system, including the acquisition layer, analysis layer, decision layer and response layer design, restoration layer, it also includes communication services, upgrade services, configuration services and other public management module. Based on the theoretical model of immune evolution, an artificial immune theory model of public opinion and crisis warning with adaptive dynamic evolution ability is developed.

\section{B. Prototype design of artificial immune system for trend forecast of new media public opinions and early warning of crisis}

Taking Weibo as an example, it collects and discovers hot topics as public opinion data through new media environment to analyze, predicts according to established models and thresholds, and forms a perfect platform for the evolution of public opinion to provide managers with media opinion on specific network events early warning of development trend. The main collection targets of the system are the forwarding volume of the hot topics in Weibo, original Weibo with identifiers, and hot topics with high comments. Through the application program interface provided by Sina for continuous Weibo's data collection, get the public opinion information using the Chinese Academy of Sciences word segmentation system and text clustering, and then establish the public opinion keyword library, constitute the public opinion keyword library, set weights and thresholds for each type of public opinion as the input of public opinion early warning model, and finally get the grading value of early warning that can get early warning results comparing with the public opinion keyword which play a role as crisis immunity.

\section{THEORETICAL MODEL AND DESIGN PLAN}

\section{A. Simulate human immune principle and construct a theoretical model of multi-layer public opinion evolution and crisis defense}

The theoretical model aims to build a variety of existing public opinion monitoring components into a multi-layered system of public opinion evolution to form a comprehensive and multi-level stereoscopic monitoring and defense system for new media systems (such as Weibo platform).The model includes the following five layers:

(1) The first layer is the acquisition layer, including the new media search engine, data cleaning, filtering, extraction, vocabulary analysis module to form a sensitive word scanning and shielding subsystem. The main task is to actively search and record and shield sensitive information. They are typical traditional static defense technologies, which can find out and resist most of the malicious public opinions and greatly raise the technical threshold that triggers the public opinion crisis.

(2) The second layer is the analysis layer. It consists of modules such as sensitive word detection, information mining and public opinion inducement traps. The active detection system is formed through the dynamic matching of antibody gene library (malicious opinion hot words) to detect malicious public opinions in time and release early warning of public opinion crisis.

(3) The third layer is the response layer, including automatic response and manual response. This layer including public opinion clustering, positive public opinion guidance and public opinion confrontation and other technical means. The main task is respond to negative information according to the malicious public opinion propensity and public opinion crisis alert.

(4) The fourth layer is the disposal layer, which consists of technical and artificial means such as disposal decision support module, equipment redundancy and disposal of resource base. In the case of hot topics under the guidance of malicious public opinion, try to provide the required positive public opinion release and guidance service through quick disposition, and provide downgrading services (deleting or blocking) if necessary, and protect the integrity of positive public opinion information.

(5) The fifth layer is the recovery layer, which consists of modules such as system backup, crisis removal and public opinion restoration. In the remission period of public opinion, the backup system backs up the pivotal information of public opinion in order to deeply analyze and guide the follow-up crisis clearance process. The public opinion recovery system uses the backup information to restore the positive public opinion information.

Multi-layer defense system will make full use of the existing variety of public opinion monitoring technology for organic integration, so as to maximize the ability of crisis prevention, and in accordance with the state of the crisis, the effective use of response and automatic disposal technology so that public opinion crisis reduced to a minimum .

The final results of the defense system can be achieved as follows: (1)The first three layers of public opinion information can be automatically predicted, responded and cleared promptly; (2)The negative information that reaches the fourth level starts early warning and provides decision support for disposal, which will lead to serious public opinion crisis ; (3)Public opinion crisis through the fourth layer will start the emergency state, make the system gradually recover from the crisis through human intervention, to avoid the harm from expanding.

\section{B. Prototype design and Empirical Study of immune system for Weibo public opinion crisis warning}

Public opinion prediction strategy is come forward according to the analysis of new media environment under the weibo application platform; Combining Chinese lexical analysis system of Chinese Academy of Sciences for the word segmentation and learning the K-means text clustering 
algorithm and taking the weibo's popular topic public opinion discovery as the application platform to improve it, which can achieve high accuracy of public opinion discovery of weibo's hot topic; constructing the BP the neural network model and combining the genetic algorithm with simulated annealing algorithm to optimize the BP neural network model. Aiming at the application platform of Weibo's hot topic, prototype software suitable for the prediction of public opinion trend of Weibo is proposed.

The research take Weibo as an empirical environment, establish a public opinion trend warning platform, discover popular topics in time by using the ICTCLAS Chinese Segmentation System of the Chinese Academy of Sciences, and then conduct empirical research on public opinion trend forecasting and simulated early warning experiments. The first two phases of the research results and prototype systems and other related technologies in accordance with the evolution of the hierarchical structure of the media were integrated to achieve public opinion monitoring system platform based on immune mechanism, besides, run and perfect the evolution of public opinion prototype system prototype.

\section{SUMMARY}

The biological immune system is a virus (antigen) detection and control system essential for organisms, especially humans. It consists of organs, tissues, antibody cells, immune effector molecules and related immune genes with immune function. Healthy individuals effectively protect themselves against pathogenic factors (antigens) such as external pathogens, harmful foreign bodies and their own cancerous cells by immune response, immune regulation, immune memory and immune surveillance mechanisms and immune evolution of immune system. The immune system includes nonspecific immunity and specific immunity. Specific immunity is a kind of adaptive complex system with intelligence and evolutionary ability, which is formed by the resistance of healthy individuals gradually accumulating when they resist the invasion of new germs. In recent years, the artificial immune system inspired by biological immune mechanism has become a hot research frontier, and its application has covered many fields such as network prevention and control, big data analysis, pattern recognition, fault diagnosis and evolutionary computation.

In the new media environment, social emergencies often stimulate the surging situation of online public opinion, thus affecting the stability and development of society. The new media represented by Weibo and WeChat has fundamentally changed the media ecological structure. The major and sudden hot events of public opinion in the Beijing-Tianjin-Hebei integration environment will lead to sudden changes in online public opinion and set off a wave. Since the self-media has the advantages of instant, convenience, widespread usage and strong interactivity that other traditional media cannot match which, to a great extent, poses a serious challenge to social public security while bringing security risks of internet virtual society under the background of new media.

Compared with the traditional media, the important feature of the new media is the interactive of communication mode, which can prompt public opinion to rise rapidly. The government departments and relevant operator must rely on and grasp the law of the development and evolution of new media consensus, guide public opinion timely and establish effective crisis response and disposal mechanisms according to the specific conditions in case the situation deteriorate dramatically. Therefore, the existing public opinion monitoring system must reflect the dynamic process of decision-making, response and recovery on the basis of static collection and analysis. And then form the multi-layer immune defense system.

On the basis of the new media environment and artificial immune principle, this research studies and establishes adaptive dynamic evolution model of Internet public opinion, designs and implements an effective public opinion monitoring and prevention and control system prototype, and then uses the intelligent algorithm and program design to develop public opinion monitoring and dynamic prevention and control system software. This kind of practical system with adaptive active monitoring and dynamic emergency response and other immune functions will have broad application prospects in the face of the major and sudden hot events of public opinion in the Beijing-Tianjin-Hebei integration environment.

This paper is supported by the Beijing Humanities and Social Science Research Fund Project (15ZHA004), hereby thanks.

\section{REFERENCES}

[1] Yinfeng Zhang, The New Orientation of Public Opinion Research under the Background of New Media, Theory And Modernization, 2012.4.

[2] Yuexin Lan, Xilin Dong, Chengxin Chen, Research on Local Governments to Deal with Network of Public Opinion Crisis Warning, Research on Local Governments to Deal with Network of Public Opinion Crisis Warning, Journal of Modern Information, 2012.5.

[3] Yiwen Zhang, Research on the Mechanism of Public Opinion on Internet for Unexpected Emergency, Doctoral Dissertation of Beijing University of Posts and Telecommunications, 2012.

[4] Lina Zhou, Study on the Topic Detection Function of Intelligent Monitoring System for Network Public Opinion, Science and Technology Information Development and Economy, 2010.8.

[5] P. M. Lydyard, etc. Instant Notes in Immunology, BIOS Scientific Published Limited, 2010.

[6] Hongwei MO, Xingquan ZUO. Artificial Immune System, Beijing: Science Press, 2009.

[7] Licheng Jiao. Immune Optimization--Computation Learning and Recognition, Beijing: Science Press, 2005. 